\title{
Baseline diabetes as a way to predict CV outcomes in a lipid-modifying trial: a meta-analysis of 330,376 patients from 47 landmark studies
}

\author{
Michel P. Hermans ${ }^{1 *}$, Evariste Bouenizabila², Daniel K. Amoussou-guenou ${ }^{3}$, Sylvie A. Ahn ${ }^{4}$ and Michel F. Rousseau ${ }^{4}$
}

\begin{abstract}
Background: Diabetes is a major cardiovascular risk factor. However, its influence on the rate of occurrence of cardiovascular (CV) events during a clinical trial that included a diabetes subgroup has not yet been quantified.

Aims: To establish equations relating baseline diabetes prevalence and incident CV events, based on comparator arms data of major lipid-modifying trials.

Methods: Meta-analysis of primary outcomes (PO) rates of key prospective trials, for which the baseline proportion of diabetics was reported, including studies having specifically reported CV outcomes within their diabetic subgroups.

Results: 47 studies, representing 330,376 patients (among whom 124,115 diabetics), were analyzed as regards the relationship between $\mathrm{CV}$ outcomes rates (including CHD) and the number of diabetics enrolled. Altogether, a total of 18,445 and 16,156 events occurred in the comparator and treatment arms, respectively. There were significant linear relationships between diabetes prevalence and both $P O$ and $C H D$ rates $(\% / y e a r): y=0.0299^{*} x+3.12[P O](p=0.0128)$; and $y=0.0531^{*} x+1.54[C H D](p=0.0094)$, baseline diabetes predicting PO rates between $3.12 \% / y e a r$ (no diabetic included) and $6.11 \% /$ year (all patients diabetic); and CHD rates between $1.54 \% /$ year (no diabetic) and $6.85 \% / y e a r$ (all patients diabetic). The slopes of the equations did not differ according to whether they were derived from primary or secondary prevention trials.

Conclusions: Absolute and relative CV risk associated with diabetes at inclusion can be readily predicted using linear equations relating diabetes prevalence to primary outcomes or CHD rates.
\end{abstract}

Keywords: Diabetes, Cardiovascular, Coronary heart disease, Clinical trial, Residual risk, Lipids

\section{Introduction}

Key prospective trials have demonstrated the effectiveness of long-term control of conventional risk factors (RFs) to prevent cardiovascular (CV) events. Next to decreasing tobacco use and physical inactivity, indisputable gains were achieved by targeting hypertension and hypercholesterolemia. Nevertheless, there remained a high residual risk of incident $\mathrm{CV}$ events in control and comparator arms of these trials, even in patients receiving appropriate standard of care [1-4]. This residual risk

\footnotetext{
*Correspondence: michel.hermans@uclouvain.be

'Division of Endocrinology \& Nutrition, Cliniques universitaires St-Luc and Institut de Recherche Expérimentale et Clinique (IREC), Université catholique de Louvain, Brussels, Belgium

Full list of author information is available at the end of the article
}

is driven by non-modifiable RFs (age; gender; familial or genetic features; and diabetes) and by modifiable conventional or emerging RFs (eg. atherogenic dyslipidemia; remnant lipoproteins; hyperglycaemia; hyperinsulinaemia; metabolic syndrome; subclinical inflammation; and chronic kidney disease).

Based on epidemiology and prospective studies, type 2 diabetes mellitus (T2DM) significantly increases the absolute risk of developing coronary heart disease (CHD), and confers a higher residual risk of large and small vessel damage. In the microcirculation, such risk is directly related to hyperglycaemia, whereas in large vessels, this residual risk is linked to hypertension, low-density lipoproteins (LDL); non-LDL dyslipidemias; and other metabolic comorbidities [5-10]. As a result, having T2DM, either individually or at 
a sub-group level (within a cohort or population) increases residual CV risk to an extent that needs to be determined. Since residual risk varies considerably from one study to another, such an evaluation would require going beyond comparing $\mathrm{CV}$ outcomes rates in diabetic vs. nondiabetic subgroups of individual trials.

The aim of this work was to establish equations relating baseline diabetes prevalence and incident $\mathrm{CV}$ events, based on comparator arms data of major clinical trials having investigated the potential $\mathrm{CV}$ benefit of various pharmacological or dietary interventions targeting, in the vast majority, lipids and lipoproteins. We performed a systematic meta-analysis of $\mathrm{CV}$ outcomes rates of those key prospective studies, for which the baseline proportion of diabetics was reported and, where available, studies having reported CV outcomes of diabetic subgroups [11-90] (Table 1).

\section{Patients and methods}

To be selected for inclusion, major clinical trials with $\mathrm{CV}$ outcomes had to meet three requirements: $(i)$ the main purpose of the trial was to study the effect on $\mathrm{CHD}$ of a pharmacological or dietary intervention targeting lipids or lipoproteins, with $\mathrm{CHD}$ rates as sole primary outcome (PO), or with a major adverse $\mathrm{CV}$ event (MACE) composite PO comprising CHD; (ii) to focus exclusively on diabetic patients, or (iii) to report data on a sufficient number of diabetic patients from pre-/posthoc analyses of DM subgroups of the main trial. Among studies conducted non-exclusively in DM patients, eligible trials had to comply with $\geq 1$ of the following criteria: $(i)$ the main trial had a subgroup of patients already diagnosed with DM at baseline, whose proportion was deemed sufficiently representative (>15\%); or (ii) the trial enrolled at least $100 \mathrm{DM}$ patients, regardless of on-study new-onset diabetes.

For each study, the following items were analyzed: $\mathrm{CV}$ risk category at baseline (primary prevention [PP], secondary prevention [SP] or mixed [PP-SP]); number of patients included; number and proportion of patients with DM at baseline; number of patients in the active or comparator arms; duration of follow-up; age at inclusion; number of males; $\mathrm{DM}$ type and duration; $\mathrm{HbA}_{1 c}$; total cholesterol (TC); low-density lipoprotein cholesterol (LDL-C); highdensity lipoprotein cholesterol (HDL-C); non-HDLcholesterol (non-HDL-C); apolipoprotein $\mathrm{B}_{100}$ (apoB); triglycerides (TG); type of pharmacological or dietary intervention; primary trial outcome; CHD outcomes (see Table 2 for CV outcomes categories); and CV events number and rates for each trial.

Results are presented as means $( \pm 1$ standard deviation (SD)), or as proportions (\%), with between-study range [BSR] described when needed. Linear regression was computed using the least-squares method. Results were considered statistically significant or non-significant (NS) for $\mathrm{p}<0.05$ or $\mathrm{p} \geq 0.05$, respectively.

\section{Results}

Forty-seven studies were selected based on the criteria defined above [11-90]. They accounted for a total of 330,376 patients. The median year of publication for all studies was 2005. Table 1 describes, for each study, the acronym's definition; the CV prevention category; the cohort size and the number or proportion of diabetic at baseline; the number of patients randomized in the active or comparator arms; the follow-up duration; and publication year. For all studies, mean age (1SD) was 61.7 (6.4) years, and the proportion of males was 74 (17) \%. Regarding ethnicity, the majority of patients studied were Caucasian (median $86.5 \%$ [between-study range (BSR 0 \%)-99.2 \%] Three studies [JELIS; MEGA; and PROFIT-J] included only Japanese patients [59, 66, 74]. Among studies, 8 of 47 (17\%; $n=42,279)$ enrolled patients in PP at baseline; 17 of 47 (36 \%; $n=131,425)$ included populations whose CV risk was a mix of PP and SP; and 22 of 47 (47 \%; $n=156,672)$ were SP trials. Lipid values at baseline were (mg/dL): 209 (34) [TC]; 126 (32) [LDL-C]; 44 (7) [HDL-C]; 161 (32) [non-HDL-C]; 99 (19) [apoB] and 162 (27) [TG]. In total, these studies have included 124,115 diabetic patients, representing $42.1 \%$ [BSR $2.3 \%-100 \%$ ] of the population studied. For studies that reported diabetes duration, it averaged 7.5 (4.9) years, whereas metabolic control assessed by $\mathrm{HbA}_{1 \mathrm{c}}$ was 7.49 (0.68) \% (Table 3). The trials investigated the following interventions over a mean (1SD) duration of 4.4 (1.9) years [BSR: 1.0-13.3 years]: statins (21 trials); fibrates (9 trials); n-3 fatty acids and/or traditional Mediterranean diet (5 trials); niacin (4 trials); CETP-inhibitor (2 trials); PPAR- $\gamma$ agonist (2 trials); ezetimibe (1 trial); PPAR- $\alpha / \gamma$ agonist (1 trial); and Lp-PLA2 inhibitor (1 trial) (Table 4).

For all 47 studies, a total of 18,445 and 16,156 events occurred in the comparator and treatment arms, respectively. On an annual basis, this was equivalent to an average rate of occurrence for the primary $\mathrm{CV}$ outcome of 3.6 (2.4) \%/year [BSR 0.5-11.8] (comparator) and 3.0 (1.9)\%/year [BSR 0.3-9.1] (treatment), respectively (Table 4). The slopes of the equations relating PO rates $(y)$ to diabetes prevalence (x) did not differ according to whether they were derived from PP or SP trials: thus, for PP trials $y=0.0208^{*} \mathrm{x}+0.53$ $\left(\mathrm{R}^{2}=0.6369 ; p=0.0058\right)$, whereas $\mathrm{y}=0.0267^{*} \mathrm{x}+3.76$ $\left(\mathrm{R}^{2}=0.1436 ; p=0.0464\right)$ for SP trials.

When comparing PO rates from the comparator arms of studies published prior to $2005 v s$. those published $\geq 2005$, average PO incidence decreased from $3.7 \% /$ year $[<2005]$ to $2.7 \% /$ year [ 22005$]$ for non-diabetic patients, ie. absolute and relative reductions of $1 \%$ and $28 \%$ (NS). For diabetic patients, the event rate decreased from $5.0 \%$ year $[<2005]$ 
Table 1 Overview of 47 landmark prospective clinical trials with CV outcomes having included a substantial number and/or proportion of diabetic patients at baseline

\begin{tabular}{|c|c|c|c|c|c|c|c|c|c|}
\hline & CV prevention & $\begin{array}{l}\text { Patients } \\
n\end{array}$ & $\begin{array}{l}\text { Diabetes } \\
n\end{array}$ & $\%$ & $\begin{array}{l}\text { Active arm } \\
n\end{array}$ & $\begin{array}{l}\text { Comparator arm } \\
n\end{array}$ & $\begin{array}{l}\text { Follow-up } \\
\text { years }\end{array}$ & Publication year & Reference \\
\hline $4 \mathrm{D}$ & PP-SP & 1255 & 1255 & 100 & 619 & 636 & 4.0 & 2005 & [11] \\
\hline $4 S$ & $\mathrm{SP}$ & 4444 & 202 & 5 & 2221 & 2223 & 5.4 & 1994 & {$[12-14]$} \\
\hline diabetes substudy & $\mathrm{SP}$ & 202 & 202 & 100 & 105 & 97 & 5.4 & 1997 & [14] \\
\hline ACCORD-Lipid & PP-SP & 5518 & 5518 & 100 & 2765 & 2753 & 4.7 & 2010 & {$[15,16]$} \\
\hline ADDITION-Europe & PP-SP & 3055 & 3055 & 100 & 1678 & 1377 & 5.3 & 2011 & {$[17,18]$} \\
\hline AFCAPS/TexCAPS & PP & 6605 & 155 & 2 & 3304 & 3301 & 5.2 & 1998 & {$[19,20]$} \\
\hline AIM-HIGH & SP & 3414 & 1158 & 34 & 1718 & 1696 & 3.0 & 2011 & {$[21,22]$} \\
\hline AleCardio & SP & 7226 & 7226 & 100 & 3616 & 3610 & 2.0 & 2014 & {$[23,24]$} \\
\hline ALERT & PP-SP & 2102 & 396 & 19 & 1050 & 1052 & 5.1 & 2003 & {$[25]$} \\
\hline ALLHAT-LLT & PP-SP & 10355 & 3638 & 35 & 5170 & 5185 & 4.8 & 2002 & {$[26]$} \\
\hline Alpha-Omega & SP & 4837 & 1754 & 36 & 2404 & 2433 & 3.4 & 2010 & [27] \\
\hline ASCOT-LLA & PP & 10305 & 2532 & 25 & 5168 & 5137 & 3.3 & 2003 & {$[28,29]$} \\
\hline diabetes substudy & PP & 2532 & 2532 & 100 & 1258 & 1274 & 3.3 & 2005 & [29] \\
\hline ASPEN & PP & 2410 & 2410 & 100 & 1211 & 1199 & 4.0 & 2006 & [30] \\
\hline AURORA & PP-SP & 2773 & 731 & 26 & 1389 & 1384 & 3.8 & 2009 & {$[31,32]$} \\
\hline diabetes substudy & PP-SP & 731 & 731 & 100 & 388 & 343 & 2.8 & 2011 & {$[32]$} \\
\hline $\mathrm{BIP}$ & SP & 3090 & 309 & 10 & 1548 & 1542 & 6.2 & 2000 & {$[33,34]$} \\
\hline CARDS & PP & 2838 & 2838 & 100 & 1428 & 1410 & 3.9 & 2004 & {$[35]$} \\
\hline CARE & SP & 4159 & 586 & 14 & 2081 & 2078 & 5.0 & 1998 & [36-38] \\
\hline diabetes substudy & SP & 586 & 586 & 100 & 282 & 304 & 5.0 & 1998 & [38] \\
\hline CDP (clofibrate) & SP & 3892 & 1517 & 39 & 1103 & 2789 & 6.2 & 1975 & {$[39,40]$} \\
\hline CDP (niacin) & $\mathrm{SP}$ & 3908 & 1524 & 39 & 1119 & 2789 & 6.2 & 1975 & {$[39,40]$} \\
\hline dal-OUTCOMES & SP & 15871 & 3882 & 24 & 7938 & 7933 & 2.6 & 2012 & {$[41,42]$} \\
\hline DIS & PP & 761 & 761 & 100 & 379 & 382 & 5.0 & 1991 & {$[43]$} \\
\hline FIELD & PP-SP & 9795 & 9795 & 100 & 4895 & 4900 & 5.0 & 2005 & [44-46] \\
\hline GISSI-Prevenzione & SP & 4271 & 582 & 14 & 2138 & 2133 & 2.0 & 2000 & [47] \\
\hline GREACE & SP & 1600 & 313 & 20 & 880 & 720 & 3.0 & 2002 & {$[48,49]$} \\
\hline diabetes substudy & SP & 313 & 313 & 100 & 161 & 152 & 3.0 & 2003 & [49] \\
\hline HATS & $\mathrm{SP}$ & 107 & 17 & 16 & 73 & 34 & 3.0 & 2001 & {$[50]$} \\
\hline $\mathrm{HHS}$ & PP & 4081 & 108 & 3 & 2051 & 2030 & 5.0 & 1987 & {$[51,52]$} \\
\hline diabetes substudy & PP & 135 & 135 & 100 & 59 & 76 & 5.0 & 1992 & {$[52]$} \\
\hline $\mathrm{HPS}-\mathrm{MRC} / \mathrm{BHF}$ & PP-SP & 20536 & 5963 & 29 & 10269 & 10267 & 5.0 & 2002 & {$[53,54]$} \\
\hline diabetes substudy & PP-SP & 5963 & 5963 & 100 & 2978 & 2985 & 4.8 & 2003 & {$[54]$} \\
\hline HPS2-THRIVE & SP & 25673 & 8299 & 32 & 12838 & 12835 & 3.9 & 2013 & {$[55]$} \\
\hline IDEAL & SP & 8888 & 1057 & 12 & 4439 & 4449 & 4.8 & 2005 & {$[56,57]$} \\
\hline ILLUMINATE & PP-SP & 15067 & 6661 & 44.2 & 7533 & 7534 & 1.0 & 2007 & [58] \\
\hline JELIS & PP-SP & 18645 & 3040 & 16.3 & 9326 & 9319 & 4.6 & 2007 & [59] \\
\hline LEADER & PP-SP & 1568 & 268 & 17 & 783 & 785 & 4.6 & 2002 & {$[60,61]$} \\
\hline LIPID & SP & 9014 & 782 & 9 & 4512 & 4502 & 6.1 & 1998 & [62-64] \\
\hline LIPS & $S P$ & 1677 & 202 & 12 & 844 & 833 & 3.9 & 2002 & [65] \\
\hline MEGA & PP & 7832 & 1632 & 21 & 3866 & 3966 & 5.3 & 2006 & [66] \\
\hline
\end{tabular}


Table 1 Overview of 47 landmark prospective clinical trials with CV outcomes having included a substantial number and/or proportion of diabetic patients at baseline (Continued)

\begin{tabular}{|c|c|c|c|c|c|c|c|c|c|}
\hline ORIGIN & PP-SP & 12536 & 11081 & 88.4 & 6281 & 6255 & 6.2 & 2012 & {$[67]$} \\
\hline PERFORM & SP & 19120 & 5299 & 27.7 & 9562 & 9558 & 2.4 & 2011 & [68] \\
\hline Post-CABG & $\mathrm{SP}$ & 1351 & 116 & 9 & 676 & 675 & 7.5 & 2000 & {$[69,70]$} \\
\hline PREDIMED & PP & 7447 & 3614 & 49 & 4997 & 2450 & 4.5 & 2013 & {$[71]$} \\
\hline PROACTIVE & SP & 5238 & 5238 & 100 & 2605 & 2633 & 2.9 & 2005 & {$[72,73]$} \\
\hline PROFIT-J & PP-SP & 481 & 481 & 100 & 234 & 247 & 1.8 & 2014 & {$[74]$} \\
\hline PROSPER & PP-SP & 5804 & 623 & 11 & 2891 & 2913 & 3.2 & 2002 & {$[75]$} \\
\hline RPS & PP-SP & 12505 & 7494 & 60 & 6239 & 6266 & 5.0 & 2013 & {$[76,77]$} \\
\hline SHARP & PP-SP & 9270 & 2094 & 23 & 4650 & 4620 & 4.9 & 2011 & [78] \\
\hline STABILITY & $\mathrm{SP}$ & 15828 & 5351 & 34 & 7924 & 7904 & 3.7 & 2014 & {$[79,80]$} \\
\hline STENO-2 & PP-SP & 160 & 160 & 100 & 80 & 80 & 13.3 & 2008 & {$[81]$} \\
\hline diabetes substudy & $\mathrm{SP}$ & 1501 & 1501 & 100 & 753 & 748 & 4.9 & 2006 & {$[86]$} \\
\hline VA Cooperative Study & $\mathrm{SP}$ & 532 & 128 & 24 & 268 & 264 & 1.8 & 1973 & {$[87]$} \\
\hline VA-HIT & SP & 2531 & 769 & 30 & 1264 & 1267 & 5.1 & 1999 & [88-90] \\
\hline diabetes substudy & $\mathrm{SP}$ & 769 & 769 & 100 & 377 & 392 & 5.1 & 2002 & {$[90]$} \\
\hline Total (n) & & 330376 & 124115 & & 165022 & 165354 & & & \\
\hline Mean & & & & & & & 4.4 & & \\
\hline
\end{tabular}

CV: cardiovascular; PP and SP: primary and secondary prevention. Acronyms: 4D: Die Deutsche Diabetes Dialyse studie; 4S: Scandinavian Simvastatin Survival Study; ACCORD-Lipid: Action to Control Cardiovascular Risk in Diabetes - Lipid arm; ADDITION-Europe: Anglo-Danish-Dutch Study of Intensive Treatment in People with Screen Detected Diabetes in Primary Care; AFCAPS/TexCAPS: Air Force/Texas Coronary Atherosclerosis Prevention Study; AIM-HIGH: Atherothrombosis Intervention in Metabolic Syndrome with Low HDL/High Triglycerides: Impact on Global Health Outcomes; AleCardio: A Safety and Efficacy Study to Evaluate the Potential of Aleglitazar to Reduce CV Risk in CHD Patients with a Recent ACS and T2DM; ALERT: Assessment of Lescol in Renal Transplantation; ALLHAT-LLT: Antihypertensive and Lipid-Lowering treatment to prevent Heart Attack Trial; ASCOT-LLA: Anglo-Scandinavian Cardiac Outcomes Trial - Lipid Lowering Arm; ASPEN: Atorvastatin as Prevention of CHD Endpoints in patients with Non-insulin dependent diabetes mellitus; AURORA: A Study to Evaluate the Use of Rosuvastatin in Subjects on Regular Hemodialysis: an Assessment of Survival and Cardiovascular Events; BIP: Bezafibrate Infarction Prevention; CARDS: Collaborative Atorvastatin Diabetes Study; CARE : Cholesterol and Recurrent Events; CDP: Coronary Drug Project; dal-OUTCOMES: Efficacy and safety of dalcetrapib in patients with recent acute coronary syndrome; DIS: Diabetes Intervention Study; FIELD: Fenofibrate Intervention and Event Lowering in Diabetes; GISSI-Prevenzione: Gruppo Italiano per lo Studio della Sopravvivenza nell'Infarto miocardico - Prevenzione; GREACE: Greek Atorvastatin and Coronary-heart-disease Evaluation; HATS: HDL-Atherosclerosis Treatment Study; HHS: Helsinki Heart Study; HPS - MRC/BHF: Medical Research Council and British Heart Foundation Heart Protection Study; HPS2-THRIVE: Heart Protection Study - Treatment of HDL to Reduce the Incidence of Vascular Events; IDEAL: Incremental Decrease in End Points Through Aggressive Lipid Lowering Trial; ILLUMINATE: Investigation of Lipid Level Management to Understand its Impact in Atherosclerosis Events; JELIS: Japan EPA Lipid Intervention Study; LEADER: Lower Extremity Arterial Disease Event Reduction; LIPID: Long-term Intervention with Pravastatin in Ischaemic Disease; LIPS: Lescol Intervention Prevention Study; MEGA: Primary Prevention of Cardiovascular Disease with Pravastatin in Japan; ORIGIN: Outcome Reduction with an Initial Glarigine Intervention; PERFORM: Prevention of cerebrovascular and cardiovascular Events of ischaemic origin with teRutroban in patients with a history oF ischaemic strOke or tRansient ischaeMic attack; Post-CABG (FU): Post Coronary Artery Bypass Graft Trial (follow-up); PREDIMED: Prevencion con Dieta Mediterranea; PROACTIVE: PROspective pioglitAzone Clinical Trial In macroVascular Events; PROFIT-J: PRimary preventiOn oF hlgh risk Type 2 diabetes in Japan; PROSPER: Prospective Study of Pravastatin in the Elderly at Risk; RPS: Risk and Prevention Study; SHARP: Study of Heart and Renal Protection; STABILITY: STabilization of Atherosclerotic plaque By Initiation of darapLadlb TherapY; STENO-2: STENO-2 Study; TNT: Treating to New Targets; VA Cooperative Study: Veteran Administration Cooperative Study of Atherosclerosis, Neurology Section; VA-HIT: Veterans Affairs High-Density Lipoprotein Intervention Trial

to $4.3 \%$ /year [ $\geq 2005]$, ie. absolute and relative reductions of $0.7 \%$ and $14 \%$ (NS).

Among these, 33 trials, totaling 259,151 patients, are described below as predominantly non-diabetes studies [12-14, 19-22, 25-29, 31-34, 36-42, 47-66, 68-70, 75, 78-80, 82-90] (Table 1). The mean age was 61.4 (5.5) years [BSR 47.0-75.0], and the proportion of males was 78.6 (17.8) \% [BSR 31.4-100]. Among predominantly non-diabetes studies, 4 of 33 (12\%) enrolled patients who were in PP at baseline; 9 of 33 (27\%) included mixed populations whose CV risk was either PP or SP; and 20 of 33 (61\%) were clinical trials in SP only. Lipid values at baseline were (mg/dL): 212 (38) [TC]; 129 (36) [LDL-C]; 44 (7) [HDL-C]; 165 (36) [non-HDL-C]; 98 (21) $[\mathrm{apoB}]$ and 160 (25) [TG]. In total, these studies have included 63.189 diabetic patients, representing $21.3 \%$ [BSR $2.3 \%-44.2 \%$ ] of the population studied (Table 1; Table 3). These predominantly non-diabetes studies investigated the following interventions over a mean (1SD) duration of 4.3 (1.5) years [BSR: 1.07.5 years]: statins (19 trials); fibrates (6 trials); n-3 fatty acids ( 2 trials); niacin ( 4 trials); CETP-inhibitor (2 trials); ezetimibe (1 trial); and Lp-PLA2 inhibitor (1 trial) (Table 4).

Amongst predominantly non-diabetes studies, we identified 9 diabetes sub-studies (DSS), numbering 12,732 patients, published as pre-/post-hoc sub-group analyses of DM patients $[14,29,32,38,49,52,54,86,90]$ (Table 1). The mean age was 60.4 (5.3) years [BSR 49.065.0], and the proportion of males was 74.9 (12.8) \% 
Table 2 CV outcomes categories

\begin{tabular}{|c|c|}
\hline Total mortality & all-cause death \\
\hline \multirow[t]{3}{*}{ Composite } & all CV events (including procedures) \\
\hline & MACE \\
\hline & CV death \\
\hline \multirow[t]{13}{*}{ Cardiac } & total $\mathrm{CHD} /$ major coronary events \\
\hline & nonfatal CHD \\
\hline & cardiac death/fatal CHD \\
\hline & ACS/ACE \\
\hline & all Ml \\
\hline & nonfatal Ml \\
\hline & fatal Ml \\
\hline & unstable/hospitalization-requiring AP \\
\hline & coronary revascularization ( $\mathrm{PCl}$ or $\mathrm{CABG})$ \\
\hline & life-threatening arrhytmias \\
\hline & resuscitation for cardiac arrest \\
\hline & sudden death \\
\hline & $\mathrm{CHF}$ \\
\hline Coronary imaging & $\begin{array}{l}\text { angiographic } C A D \text { progression/change in } \\
\text { coronary atheroma volume }\end{array}$ \\
\hline \multirow[t]{5}{*}{ Cerebrovascular } & all major cerebrovascular events \\
\hline & all stroke/TIA \\
\hline & nonfatal stroke \\
\hline & fatal stroke \\
\hline & carotid revascularization \\
\hline Other composite & non-CHD MACE \\
\hline Other mortality & non-CHD CV death \\
\hline Peripheral & $\begin{array}{l}\text { any PAD event (including revascularization } \\
\text { and leg amputation) }\end{array}$ \\
\hline
\end{tabular}

ACE/ACS: acute coronary event/syndrome; AP angina pectoris; CABG: coronary artery bypass graft; $C A D$ : coronary artery disease; $C H D$ : coronary heart disease; CHF: congestive heart failure; CV: cardiovascular; MACE: major adverse cardiovascular event; MI myocardial infraction; PAD: peripheral arterial disease; PCl: percutaneous coronary intervention; TIA:transient is chemic attack (adapted from [91])

[BSR 56.2-100]. Within DSS, 2 of 9 (22\%) enrolled patients who were in PP at baseline; 2 of $9(22 \%)$ included mixed populations whose CV risk was either PP or SP; and 5 of 9 (56\%) were clinical trials in SP only. Lipid values at baseline were (mg/dL): 219 (45) [TC]; 140 (41) [LDL-C]; 41 (5) [HDL-C]; 178 (44) [non-HDL-C]; and 181 (25) [TG] (Table 3). The DSS have investigated the following interventions over a mean (1SD) duration of 4.4 (1.0) years [BSR: $2.8-5.4$ years]: statins (7 trials); and fibrates (2 trials) (Table 4).

Fourteen other trials, totaling 71,225 patients, dealt exclusively with DM patients, or included a very-high proportion $(>45 \%)$ of DM patients at baseline [11, 15-18, $23,24,30,35,43-46,67,71-74,76,77,81]$, and are described below as studies focusing on diabetes (Table 1). The mean age was 62.6 (8.2) years [BSR 46.0-85.0], and the proportion of males was 63.0 (8.3) \% [BSR 42.574.4]. Mean diabetes duration was 7.5 (4.9) years [BSR 0-18.0], and HbA1 7.6 (0.7) \% [BSR 6.7-8.6] (Table 3).

Among studies focusing on diabetes, 4 of 14 (29\%) enrolled patients who were in PP at baseline; 8 of 14 (57\%) included mixed populations whose CV risk was either PP or SP; and 2 of 14 (14\%) were clinical trials in SP only. Lipid values at baseline were (mg/dL): 200 (19) [TC]; 118 (16) [LDL-C]; 46 (6) [HDL-C]; 154 (19) [nonHDL-C]; and 165 (32) [TG] (Table 3). The studies focusing on diabetes investigated the following interventions over a mean (1SD) duration of 4.8 (2.7) years [BSR: 1.813.3 years]: statins ( 5 trials); fibrates ( 4 trials); $n-3$ fatty acids and/or traditional Mediterranean diet (3 trials); PPAR- $\gamma$ agonist ( 2 trials); and PPAR- $\alpha / \gamma$ agonist ( 1 trial) (Table 4).

Among the 33 predominantly non-diabetic studies, a total of 14,732 and 12,604 events occurred in the comparator and treatment arms, respectively. On an annual basis, this was equivalent to an average rate of occurrence for the primary CV outcome of 3.8 (2.4) \%/year [BSR 0.5-11.8] (comparator) and 3.1 (1.8) \%/year [BSR 0.3-7.5] (treatment), respectively.

Amongst the 9 DSS, a total of 1,469 and 1,119 events occurred in the comparator and treatment arms, respectively. On an annual basis, this was equivalent to an average rate of occurrence for the primary $\mathrm{CV}$ outcome of 6.1 (3.0) \%/year [BSR 2.1-10.8] (comparator) and 4.0 (2.1) \%/year [BSR 0.7-7.8] (treatment), respectively.

Among the 14 studies focusing on diabetes, a total of 3,713 and 3,552 events occurred in the comparator and treatment arms, respectively. On an annual basis, this was equivalent to an average rate of occurrence for the primary CV outcome of 3.3 (2.5) \%/year [BSR 1.1-9.6] (comparator) and 2.9 (2.4) \%/year [BSR 0.8-9.1] (treatment), respectively.

In addition to $\mathrm{PO}$ rates, which include de facto $\mathrm{CHD}$, we also examined CHD rate as a separate outcome [Table 4 and Fig. 1 left panels]. Rates of CHD were issued for 21 trials and DSS for comparator and treatment arms, and amounted to [\%/year]: 11.1 and 7.2 [4S-DSS]; 1.3 and 0.9 [AFCAPS/TexCAPS]; 1.5 and 1.0 [ASCOTLLA]; 5.1 and 4.9 [AURORA]; 5.8 and 5.4 [BIP]; 12.0 and 9.3 [CARE-DSS]; 4.9 and 4.5 [CDP (clofibrate)]; 4.9 and 4.1 [CDP (niacin)]; 2.4 and 1.7 [HPS - MRC/BHF]; 2.6 and 2.0 [HPS - MRC/BHF-DSS]; 1.4 and 1.3 [HPS2THRIVE]; 5.0 and 4.2 [IDEAL]; 2.0 and 2.4 [ILLUMINATE]; 0.8 and 0.6 [JELIS]; 3.1 and 2.5 [LEADER]; 0.5 and 0.3 [MEGA]; 1.0 and 0.9 [SHARP]; 4.3 and 4.0 [STABILITY]; 1.7 and 1.4 [TNT]; 2.6 and 2.1 [TNTDSS]; and 1.9 and 1.7 [VA Cooperative Study] (Fig. 1; right panels).

The relationship between proportion of diabetic patients at inclusion and $\mathrm{PO}$ or $\mathrm{CHD}$ rates was inferred on 
Table 3 Baseline characteristics

\begin{tabular}{|c|c|c|c|c|c|c|c|c|c|c|c|}
\hline Study ${ }^{\S}$ & $\begin{array}{l}\text { Age } \\
\text { (years) }\end{array}$ & $\begin{array}{l}\text { Males } \\
(\%)\end{array}$ & $\begin{array}{l}\text { Diabetes type } \\
\text { (years) }\end{array}$ & duration & $\begin{array}{l}\mathrm{HbAlc} \\
(\%)\end{array}$ & $\begin{array}{l}\text { TC } \\
(\mathrm{mg} / \mathrm{dL})\end{array}$ & $\begin{array}{l}\text { LDL-C } \\
(\mathrm{mg} / \mathrm{dL})\end{array}$ & $\begin{array}{l}\mathrm{HDL}-\mathrm{C} \\
(\mathrm{mg} / \mathrm{dL})\end{array}$ & $\begin{array}{l}\text { Non-HDL-C } \\
(\mathrm{mg} / \mathrm{dL})\end{array}$ & $\begin{array}{l}\text { apoB } \\
(\mathrm{mg} / \mathrm{dL})\end{array}$ & $\begin{array}{l}\text { TG } \\
(\mathrm{mg} / \mathrm{dL})\end{array}$ \\
\hline $4 \mathrm{D}$ & 66 & 54 & T2DM & 18 & 6.7 & 218 & 125 & 36 & 182 & $\sim$ & 261 \\
\hline $4 S$ & 59 & 81 & $\sim$ & & & 260 & 188 & 46 & 214 & $\sim$ & 132 \\
\hline diabetes substudy & 60 & 78 & $\sim$ & & & 259 & 186 & 43 & 216 & $\sim$ & 150 \\
\hline ACCORD-Lipid & 62 & 69 & $\mathrm{~T} 2 \mathrm{DM}$ & 10 & 8.3 & 175 & 100 & 38 & 137 & $\sim$ & 164 \\
\hline ADDITION-Europe & 60.3 & 58 & T2DM & 0 & 7 & 214 & 133 & 46 & 168 & $\sim$ & 146 \\
\hline AFCAPS/TexCAPS & 58 & 85 & T1DM; T2DM & & & 221 & 150 & 37 & 184 & $\sim$ & 158 \\
\hline AIM-HIGH & 64 & 85 & $\sim$ & & 6.7 & 146 & 74 & 35 & 111 & 83 & 168 \\
\hline AleCardio & 60.8 & 73 & $\mathrm{~T} 2 \mathrm{DM}$ & 8.6 & 7.8 & 152 & 79 & 42 & 110 & $\sim$ & 152 \\
\hline ALERT & 50 & 66 & $\sim$ & & & 247 & 158 & 50 & 197 & $\sim$ & 195 \\
\hline ALLHAT-LLT & 66 & 51 & T2DM & & & 224 & 146 & 48 & 176 & $\sim$ & 152 \\
\hline Alpha-Omega & 69 & 78 & $\sim$ & & & 183 & 100 & 50 & 133 & $\sim$ & 146 \\
\hline ASCOT-LLA & 63 & 81 & $\sim$ & & & 212 & 131 & 50 & 162 & $\sim$ & 150 \\
\hline diabetes substudy & 63.6 & 76 & $\mathrm{~T} 2 \mathrm{DM}$ & & & 205 & 128 & 46 & 159 & $\sim$ & 168 \\
\hline ASPEN & 61 & 66 & T2DM & 8 & 7.8 & 194 & 113 & 47 & 147 & $\sim$ & 147 \\
\hline AURORA & 64 & 62 & $\sim$ & & & 176 & 100 & 45 & 131 & 82 & 157 \\
\hline diabetes substudy & 65 & 66 & $\sim$ & & & 174 & 97 & 43 & 131 & $\sim$ & 168 \\
\hline BIP & 60 & 91 & $\mathrm{~T} 2 \mathrm{DM}$ & & & 212 & 148 & 35 & 177 & $\sim$ & 145 \\
\hline CARDS & 62 & 68 & T2DM & 8 & 7.9 & 207 & 117 & 54 & 153 & 117 & 173 \\
\hline CARE & 59 & 86 & $\sim$ & & & 209 & 139 & 39 & 170 & $\sim$ & 156 \\
\hline diabetes substudy & 61 & 80 & $\sim$ & & & 206 & 136 & 38 & 168 & $\sim$ & 164 \\
\hline CDP (clofibrate) & & 100 & $\sim$ & & & 252 & $\sim$ & $\sim$ & $\sim$ & $\sim$ & 183 \\
\hline CDP (niacin) & & 100 & $\sim$ & & & 253 & $\sim$ & $\sim$ & $\sim$ & $\sim$ & 183 \\
\hline dal-OUTCOMES & 60.2 & 81 & $\sim$ & & & 145 & 76 & 42 & 103 & 81 & 134 \\
\hline DIS & 46 & 56 & $\mathrm{~T} 2 \mathrm{DM}$ & 0 & & 218 & $\sim$ & $\sim$ & $\sim$ & $\sim$ & 157 \\
\hline FIELD & 62 & 63 & $\mathrm{~T} 2 \mathrm{DM}$ & 5 & 6.9 & 195 & 119 & 43 & 152 & 97 & 173 \\
\hline GISSI-Prevenzione & 60 & 86 & $\begin{array}{l}\text { T2DM (79\%) } \\
\operatorname{T1DM}(21 \%)\end{array}$ & & & 229 & 152 & 46 & 183 & $\sim$ & 166 \\
\hline GREACE & & 79 & $\sim$ & & & 264 & 193 & 39 & 225 & $\sim$ & 159 \\
\hline diabetes substudy & 55 & 56 & $\begin{array}{l}\text { T2DM (92\%) } \\
\text { T1DM (8 \%) }\end{array}$ & 10.5 & 7.5 & 271 & 189 & 35 & 236 & $\sim$ & 221 \\
\hline HATS & 53 & 87 & $\sim$ & & & 200 & 128 & 30 & 170 & 119 & 219 \\
\hline HHS & 47 & 100 & $\sim$ & & & 270 & 189 & 47 & 223 & $\sim$ & 175 \\
\hline diabetes substudy & 49 & 100 & $\mathrm{~T} 2 \mathrm{DM}$ & 4.5 & & 292 & 200 & 46 & 246 & $\sim$ & 214 \\
\hline HPS - MRC/BHF & & 75 & $\sim$ & & & 228 & 131 & 41 & 187 & 114 & 186 \\
\hline diabetes substudy & 62.1 & 70 & $\begin{array}{l}\text { T2DM (90\%) } \\
\text { T1DM (10\%) }\end{array}$ & 27 & 7 & 220 & 124 & 41 & 179 & 110 & 204 \\
\hline HPS2-THRIVE & 64.9 & 82.7 & $\sim$ & & & 128 & 63 & 44 & 84 & 68 & 127 \\
\hline IDEAL & 62 & 81 & $\sim$ & & & 197 & 122 & 46 & 151 & 119 & 151 \\
\hline ILLUMINATE & 61.3 & 77.8 & $\mathrm{~T} 2 \mathrm{DM}$ & & & 157 & 80 & 49 & 108 & 73 & 127 \\
\hline JELIS & 61 & 31.4 & $\sim$ & & & 275 & 181 & 59 & 216 & $\sim$ & 153 \\
\hline LEADER & 68 & 100 & $\sim$ & & & 218 & 131 & 46 & 172 & $\sim$ & 213 \\
\hline LIPID & 62 & 83 & $\sim$ & & & 218 & 150 & 36 & 182 & 133 & 142 \\
\hline LIPS & 60 & 84 & T2DM; T1DM & & & 200 & 131 & 38 & 162 & $\sim$ & 160 \\
\hline MEGA & 58.3 & 32 & $\sim$ & & & 242 & 157 & 58 & 184 & $\sim$ & 128 \\
\hline
\end{tabular}


Table 3 Baseline characteristics (Continued)

\begin{tabular}{|c|c|c|c|c|c|c|c|c|c|c|c|}
\hline ORIGIN & 63.5 & 65 & $\mathrm{~T} 2 \mathrm{DM}$ & 5.4 & & 189 & 112 & 46 & 143 & $\sim$ & 142 \\
\hline PERFORM & 67.2 & 62.5 & $\sim$ & & & $\sim$ & 93 & $\sim$ & $\sim$ & & \\
\hline Post-CABG & 61.7 & 92 & $\sim$ & & & 226 & 156 & 39 & 187 & $\sim$ & 158 \\
\hline PREDIMED & 67 & 43 & $\sim$ & & & 219 & 143 & 53 & 172 & 102 & 142 \\
\hline PROACTIVE & 61.8 & 66 & $\mathrm{~T} 2 \mathrm{DM}$ & 9.5 & 8.1 & 199 & 114 & 45 & 154 & $\sim$ & 198 \\
\hline PROFIT-J & 85 & 65 & T2DM & 11.3 & 7.4 & 198 & 115 & 55 & 144 & $\sim$ & 141 \\
\hline PROSPER & 75 & 48 & $\sim$ & & & 220 & 147 & 50 & 170 & $\sim$ & 133 \\
\hline RPS & 63.9 & 61.5 & $\sim$ & & 6.7 & 216 & 132 & 51 & 165 & $\sim$ & 150 \\
\hline SHARP & 62 & 63 & $\sim$ & & & 189 & 107 & 43 & 146 & 92 & 205 \\
\hline STABILITY & 65 & 81 & $\sim$ & & & $\sim$ & 80 & 45 & $\sim$ & & \\
\hline STENO-2 & 54.9 & 74 & T2DM & 5.8 & 8.6 & 210 & 133 & 40 & 170 & $\sim$ & 159 \\
\hline TNT & 61 & 81 & $\sim$ & & & 175 & 97 & 47 & 128 & 111 & 151 \\
\hline diabetes substudy & 63 & 73 & $\sim$ & 8.5 & 7.4 & 175 & 96 & 45 & 130 & 113 & 171 \\
\hline VA Cooperative Study & 55 & 100 & $\sim$ & & & 244 & $\sim$ & $\sim$ & $\sim$ & $\sim$ & $\sim$ \\
\hline VA-HIT & 64 & 100 & $\sim$ & & & 175 & 111 & 32 & 143 & 96 & 161 \\
\hline diabetes substudy & 65 & & $\sim$ & & & 172 & 108 & 31 & 141 & $\sim$ & 166 \\
\hline mean & 61.7 & 74 & & 7.5 & 7.49 & 209 & 126 & 44 & 161 & 99 & 162 \\
\hline standard deviation & 6.4 & 17 & & 4.9 & 0.68 & 34 & 32 & 7 & 32 & 19 & 27 \\
\hline
\end{tabular}

s: see legend to Table 1 for study acronyms definition; apoB: apolipoprotein B100; C: cholesterol; HbA1c: glycated haemoglobin A1c; HDL: high-density lipoprotein; LDL: low-density lipoprotein; T1DM and T2DM: type 1 and type 2 diabetes mellitus; TG: triglycerides

the basis of the comparator and treatment arms data from the 33 predominantly non-diabetic studies, including where appropriate the rates for the corresponding DSS, ie 259,151 patients. Both for PO and CHD, there was a highly significant linear relationship between the proportion of diabetics enrolled and events rates, both in comparator arms $(\mathrm{p}=0.0128$ [PO] and $\mathrm{p}=0.0094$ [CHD]; Fig. 1; upper panels $)$ and active arms $(\mathrm{p}=0.0470$ $[\mathrm{PO}]$ and $\mathrm{p}=0.0272$ [CHD]; Fig. 1; lower panels). When comparing the slopes of the equations between PO and the proportion of diabetes at baseline in the comparator arm of studies published $<2005$ and from 2005 to 2014, they rose from 0.0129 to 0.0162 , ie a relative increase of $26 \%$ (not shown). Such relationships were more pronounced as regards CHD events, exhibiting steeper gradients than those of PO rates, with slope coefficients higher by a relative $78 \%$ [comparator arms] and $110 \%$ [treatment arms]. Vis-à-vis the comparator arms, the slopes of the relationships between proportions of diabetics and events rates in the treatment arms of the same studies were attenuated, by a relative $45 \%$ [PO rates] and 34\% [CHD events] (Fig. 1; lower panels).

Computing occurrence rates of $\mathrm{PO}$ and $\mathrm{CHD}$ in the comparator arms showed that the proportion of diabetics at inclusion predicted $\mathrm{PO}$ rates ranging from $3.12 \% /$ year (no diabetic included) to $6.11 \% /$ year (all patients diabetic). Predicted CHD rates depending on baseline diabetes prevalence ranged from $1.54 \% /$ year (no diabetic included) to $6.85 \% /$ year (all patients diabetic).
This implies that a cohort exclusively composed of diabetic patients would present a PO rate already increased by an absolute $3 \%$ /year due to the mere fact of being diabetic at baseline. Such an out-of-hand absolute increase in events rate due to the diabetic state would further increase to $5.3 \%$ /year when it comes to the risk of incident CHD (Fig. 1; upper panels).

By relating incidence rates of $\mathrm{PO}$ and $\mathrm{CHD}$ in the treatment arms, it appears that the proportion of diabetics at inclusion predicts PO rates ranging from $2.65 \% /$ year (no diabetic included) to $4.31 \% /$ year (all patients diabetic). Predicted CHD rates based on diabetes prevalence ranged from $1.64 \% /$ year (no diabetic included) to $5.13 \% /$ year (all patients diabetic). It follows that a cohort exclusively composed of diabetic patients would present an on-treatment PO rate increased by an absolute $1.7 \%$ /year solely due to the presence of DM at baseline. Such an absolute increase in events rate due to diabetes would further increase to 3.5 \%/year for incident CHD risk (Fig. 1; lower panels).

The comparison of these equations linking the proportion of diabetics and outcome rates in comparator vs. treatment arms allows for determining whether being diabetic (apart from the observation that it increases the absolute rate of occurrence of $\mathrm{CV}$ events) is associated with an idiosyncratic on-treatment clinical response. As for PO and CHD, diabetic patients were characterized by a clinical response that was better than that calculated for a non-diabetic population that would have been subject to the same therapeutic interventions. Thus, residual CV risk 
Table 4 Primary CV outcome rates in the active (treatment) and control (comparator/placebo) arms

\begin{tabular}{|c|c|c|c|c|c|c|c|c|c|c|c|}
\hline$\overline{\text { Study }}$ & Intervention & $\begin{array}{l}\text { Primary; secondary } \\
\text { CV outcomes }\end{array}$ & $\begin{array}{l}\text { Events }(\mathrm{n}) \\
\text { treatment }\end{array}$ & $\begin{array}{l}\text { Events (\%) } \\
\text { treatment }\end{array}$ & $\begin{array}{l}\text { Rate } \\
\text { (\%.year-1) } \\
\text { treatment }\end{array}$ & $\begin{array}{l}\text { Events (n) } \\
\text { control }\end{array}$ & $\begin{array}{l}\text { Events (\%) } \\
\text { control }\end{array}$ & $\begin{array}{l}\text { Rate (\%year-1) } \\
\text { control }\end{array}$ & HR & $\begin{array}{l}95 \% \mathrm{Cl} \\
\text { for } \mathrm{HR}\end{array}$ & $P$ \\
\hline $4 \mathrm{D}$ & statin & $C ; D+J$ & 226 & 36.5 & 9.13 & 243 & 38.2 & 9.55 & 0.96 & $0.77-1.1$ & 0.37 \\
\hline 45 & statin & A & 182 & 8.2 & 1.52 & 256 & 11.5 & 2.13 & 0.71 & $0.58-0.85$ & 0.0003 \\
\hline $\begin{array}{l}\text { diabetes } \\
\text { substudy }\end{array}$ & statin & A & 15 & 14.3 & 2.65 & 24 & 24.7 & 4.58 & 0.58 & NR & 0.087 \\
\hline ACCORD-Lipid & fibrate & $C_{i} J+D$ & 291 & 10.5 & 2.24 & 310 & 11.3 & 2.40 & 0.93 & $0.79-1.08$ & 0.32 \\
\hline ADDITION-Europe & statin/other & $B ; D+J+M+Z$ & 121 & 7.2 & 1.36 & 117 & 8.5 & 1.60 & 0.85 & $0.65-1.05$ & 0.12 \\
\hline AFCAPS/TexCAPS & statin & $C_{i} E$ & 116 & 3.5 & 0.68 & 183 & 5.5 & 1.07 & 0.63 & $0.50-0.79$ & $<0.001$ \\
\hline AIM-HIGH & niacin & $C ; G+J+H+M$ & 282 & 16.4 & 5.47 & 274 & 16.2 & 5.39 & 1.02 & $0.87-1.21$ & 0.8 \\
\hline AleCardio & PPAR-a/Y & $C ; D+J$ & 344 & 9.5 & 4.76 & 360 & 10.0 & 4.99 & 0.95 & $0.83-1.11$ & 0.57 \\
\hline ALERT & statin & $C ; G+J+M$ & 112 & 10.7 & 2.09 & 134 & 12.7 & 2.50 & 0.84 & $0.64-1.06$ & 0.14 \\
\hline ALLHAT-LLT & statin & A & 631 & 12.2 & 2.54 & 641 & 12.4 & 2.58 & 0.99 & $0.89-1.11$ & 0.88 \\
\hline Alpha-Omega & $\begin{array}{l}\text { n-3 fatty } \\
\text { acids }\end{array}$ & B & 336 & 14.0 & 4.11 & 335 & 13.8 & 4.05 & 1.02 & $0.87-1.17$ & 0.93 \\
\hline ASCOT-LLA & statin & $J+G$ & 100 & 1.9 & 0.59 & 154 & 3.0 & 0.91 & 0.65 & $0.50-0.83$ & 0.0005 \\
\hline $\begin{array}{l}\text { diabetes } \\
\text { substudy }\end{array}$ & statin & B & 116 & 9.2 & 2.79 & 151 & 11.9 & 3.59 & 0.78 & $0.61-0.98$ & 0.04 \\
\hline ASPEN & statin & $\begin{array}{l}C_{;} D+J+M+O \\
+L\end{array}$ & 166 & 13.7 & 3.43 & 180 & 15.0 & 3.75 & 0.91 & $0.73-1.12$ & 0.34 \\
\hline AURORA & statin & $C_{i} J+D$ & 396 & 28.5 & 7.50 & 408 & 29.5 & 7.76 & 0.97 & $0.84-1.11$ & 0.59 \\
\hline $\begin{array}{l}\text { diabetes } \\
\text { substudy }\end{array}$ & statin & $C ; G+J$ & 85 & 21.9 & 7.82 & 104 & 30.3 & 10.83 & 0.72 & $0.51-0.90$ & 0.008 \\
\hline BIP & fibrate & $C_{;} K+J+P$ & 211 & 13.6 & 2.20 & 232 & 15.0 & 2.43 & 0.91 & NR & 0.26 \\
\hline CARDS & statin & $C_{i} H+M+T$ & 83 & 5.8 & 1.49 & 127 & 9.0 & 2.31 & 0.65 & $0.48-0.83$ & 0.001 \\
\hline CARE & statin & $G+J$ & 212 & 10.2 & 2.04 & 274 & 13.2 & 2.64 & 0.77 & $0.09-0.36$ & 0.003 \\
\hline $\begin{array}{l}\text { diabetes } \\
\text { substudy }\end{array}$ & statin & $G+J+M$ & 81 & 28.7 & 5.74 & 112 & 36.8 & 7.37 & 0.78 & NR & $<0.0001$ \\
\hline CDP (clofibrate) & fibrate & A & 281 & 25.5 & 4.11 & 709 & 25.4 & 4.10 & 1.00 & NR & $N R$ \\
\hline CDP (niacin) & niacin & A & 273 & 24.4 & 3.93 & 709 & 25.4 & 4.10 & 0.96 & $0.85-1.08$ & $N R$ \\
\hline dal-OUTCOMES & $\begin{array}{l}\text { CETP } \\
\text { inhibitor }\end{array}$ & $C ; G+J+L+O$ & 656 & 8.3 & 3.20 & 633 & 8.0 & 3.09 & 1.04 & $0.93-1.16$ & 0.52 \\
\hline DIS & fibrate & $E$ & 32 & 8.4 & 1.69 & 31 & 8.1 & 1.62 & 1.04 & NR & $N R$ \\
\hline FIELD & fibrate & $C ; B+D+I+M$ & 256 & 5.2 & 1.05 & 288 & 5.9 & 1.18 & 0.89 & $0.75-1.05$ & 0.16 \\
\hline GISSI-Prevenzione & statin & $C ; A+1$ & 120 & 5.6 & 2.77 & 136 & 6.4 & 3.15 & 0.88 & $0.71-1.15$ & 0.41 \\
\hline GREACE & statin & $\begin{array}{l}C_{;} A+J+L+Q+ \\
M\end{array}$ & 112 & 12.7 & 4.24 & 180 & 25.0 & 8.33 & 0.51 & & $<0.0001$ \\
\hline $\begin{array}{l}\text { diabetes } \\
\text { substudy }\end{array}$ & statin & $\begin{array}{l}C_{i} A+J+L+Q+ \\
M\end{array}$ & 20 & 12.4 & 4.14 & 46 & 30.3 & 10.09 & 0.41 & NR & $<0.0001$ \\
\hline HATS & $\begin{array}{l}\text { statin }+ \\
\text { niacin }^{\S \S \S}\end{array}$ & $R+B ; D+J+M$ & 7 & 9.6 & 3.20 & 12 & 35.3 & 11.76 & 0.27 & NR & 0.02 \\
\hline HHS & fibrate & $C ; K+J+G$ & 56 & 2.7 & 0.55 & 84 & 4.1 & 0.83 & 0.66 & $0.08-0.53$ & $<0.02$ \\
\hline $\begin{array}{l}\text { diabetes } \\
\text { substudy }\end{array}$ & fibrate & $C ; K+J+G$ & 2 & 3.4 & 0.68 & 8 & 10.5 & 2.11 & 0.32 & NR & 0.19 \\
\hline HPS - MRC/BHF & statin & $C ; A+G$ & 1328 & 12.9 & 2.59 & 1507 & 14.7 & 2.94 & 0.88 & $0.81-0.94$ & 0.0003 \\
\hline $\begin{array}{l}\text { diabetes } \\
\text { substudy }\end{array}$ & statin & $E+B$ & 601 & 20.2 & 4.20 & 748 & 25.1 & 5.22 & 0.81 & $0.19-0.30$ & $<0.0001$ \\
\hline HPS2-THRIVE & niacin & $C ; G+M$ & 1696 & 13.2 & 3.39 & 1758 & 13.7 & 3.51 & 0.96 & $0.90-1.03$ & 0.29 \\
\hline
\end{tabular}


Table 4 Primary CV outcome rates in the active (treatment) and control (comparator/placebo) arms (Continued)

\begin{tabular}{|c|c|c|c|c|c|c|c|c|c|c|c|}
\hline IDEAL & statin & $C ; G+J+O$ & 411 & 9.3 & 1.93 & 463 & 10.4 & 2.17 & 0.89 & $0.78-1.01$ & 0.07 \\
\hline ILLUMINATE & $\begin{array}{l}\text { CETP } \\
\text { inhibitor }\end{array}$ & $C ; G+J+L$ & 464 & 6.2 & 6.16 & 373 & 5.0 & 4.95 & 1.24 & $1.09-1.44$ & 0.001 \\
\hline JELIS & $\begin{array}{l}\mathrm{n}-3 \text { fatty } \\
\text { acids }\end{array}$ & $E ; P ; I ; L ; M ; A$ & 262 & 2.8 & 0.61 & 324 & 3.5 & 0.76 & 0.81 & $0.69-0.95$ & 0.01 \\
\hline LEADER & fibrate & $\mathrm{E}$ & 150 & 19.2 & 4.95 & 160 & 20.4 & 5.20 & 0.95 & $0.76-1.21$ & 0.72 \\
\hline LIPID & statin & G & 287 & 6.4 & 1.04 & 373 & 8.3 & 1.36 & 0.77 & $0.12-0.35$ & $<0.001$ \\
\hline LIPS & statin & $C ; G+J+M$ & 181 & 21.4 & 5.50 & 222 & 26.7 & 6.83 & 0.80 & $0.64-0.95$ & 0.01 \\
\hline MEGA & statin & $C_{i} I+L+M+P$ & 66 & 1.7 & 0.32 & 101 & 2.5 & 0.48 & 0.67 & $0.49-0.91$ & 0.01 \\
\hline ORIGIN & $\begin{array}{l}\text { n-3 fatty } \\
\text { acids }\end{array}$ & $\begin{array}{l}\mathrm{D} ; \mathrm{D}+\mathrm{J}+\mathrm{U} ; \mathrm{A} ; \mathrm{l} ; \\
\mathrm{T} ; \mathrm{M}+\mathrm{W} ; \mathrm{Q} ; \mathrm{L} ; \mathrm{Z}\end{array}$ & 574 & 9.1 & 1.47 & 581 & 9.3 & 1.50 & 0.98 & $0.87-1.10$ & 0.72 \\
\hline PERFORM & antiplatelet & $D ; I$ & 1091 & 11.4 & 4.83 & 1062 & 11.1 & 4.71 & 1.03 & $0.94-1.12$ & NS \\
\hline Post-CABG & statin & $C ; D+J+M$ & 207 & 30.6 & 4.08 & 271 & 40.1 & 5.35 & 0.76 & NR & 0.04 \\
\hline PREDIMED & TMD & $C ; D+1$ & 179 & 3.6 & 0.80 & 109 & 4.4 & 1.12 & 0.71 & & \\
\hline PROACTIVE & glitazone & $C ; A+J+H+M$ & 514 & 19.7 & 6.80 & 572 & 21.7 & 7.49 & 0.91 & $0.80-1.02$ & 0.1 \\
\hline PROFIT-J & glitazone & $C ; A+J$ & 9 & 3.8 & 2.09 & 10 & 4.0 & 2.20 & 0.95 & $\begin{array}{l}0.427- \\
2.593\end{array}$ & 0.91 \\
\hline PROSPER & statin & $C ; G+J$ & 408 & 14.1 & 4.41 & 473 & 16.2 & 5.07 & 0.87 & $0.74-0.97$ & 0.01 \\
\hline RPS & $\begin{array}{l}\text { n-3 fatty } \\
\text { acids }\end{array}$ & D & 733 & 11.7 & 2.35 & 745 & 11.9 & 2.38 & 0.99 & 0.88-1.08 & 0.64 \\
\hline SHARP & $\begin{array}{l}\text { statin/ } \\
\text { ezetimibe }\end{array}$ & $C_{i} J+G+M$ & 526 & 11.3 & 2.31 & 619 & 13.4 & 2.73 & 0.84 & $0.74-0.94$ & 0.0021 \\
\hline STABILITY & $\begin{array}{l}\text { Lp-PLA2- } \\
\text { inhibitor }\end{array}$ & $C ; D+J+U$ & 769 & 9.7 & 2.62 & 819 & 10.4 & 2.80 & 0.94 & $0.85-1.03$ & 0.2 \\
\hline STENO-2 & $\begin{array}{l}\text { statin/ } \\
\text { fibrate }\end{array}$ & A & 24 & 30.0 & 2.26 & 40 & 50.0 & 3.76 & 0.60 & $0.32-0.89$ & 0.02 \\
\hline TNT & statin & $\mathrm{C} ; \mathrm{G}+\mathrm{J}+\mathrm{O}+\mathrm{T}$ & 434 & 8.7 & 1.77 & 548 & 10.9 & 2.23 & 0.79 & $0.69-0.89$ & $<0.001$ \\
\hline $\begin{array}{l}\text { diabetes } \\
\text { substudy }\end{array}$ & statin & $C_{i} G+J+O+T$ & 103 & 13.7 & 2.79 & 135 & 18.0 & 3.68 & 0.76 & $0.58-0.97$ & 0.026 \\
\hline $\begin{array}{l}\text { VA Cooperative } \\
\text { Study }\end{array}$ & fibrate & $A+B$ & 22 & 8.2 & 4.56 & 30 & 11.4 & 6.31 & 0.72 & $0.43-1.22$ & $N R$ \\
\hline VA-HIT & fibrate & $C_{i} J+G$ & 219 & 17.3 & 3.40 & 275 & 21.7 & 4.26 & 0.80 & $0.07-0.35$ & 0.006 \\
\hline $\begin{array}{l}\text { diabetes } \\
\text { substudy }\end{array}$ & fibrate & $C_{i} J+G$ & 96 & 25.5 & 4.99 & 141 & 36.0 & 7.05 & 0.71 & $0.53-0.88$ & 0.004 \\
\hline Total (n) & & & 16156 & & & 18445 & & & & & \\
\hline Mean & & & & 12.2 & 3.0 & & 14.8 & 3.6 & 0.85 & & \\
\hline
\end{tabular}

§: see legend to Table 1 for study acronyms definition; §§: see Table 2 for CV outcomes definition; §§§: \pm antioxidants; CETP: cholesteryl ester transfer protein; Cl: confidence interval; CV: cardiovascular; HR: hazard ratio; LpPLA2: lipoprotein-associated phospholipase A2; NR: not reported; NS: non significant; PPAR: peroxisome proliferator-activated receptor; TMD: traditional Mediterranean diet

persisting after treatment was further reduced in case of diabetes, in a relative proportion of $14.4 \%$ [PO] and $31.2 \%$ [CHD], respectively (Fig. 1; upper and lower panels).

\section{Discussion}

This meta-analysis shows that the presence of diabetics in a lipid-modifying trial is a determinant of $\mathrm{CV}$ events rate, the impact of which can be accurately assessed once known the proportion of diabetics enrolled, regardless of the CV risk category at baseline. Thus, the linear equations derived from this meta-analysis can be used to determine the absolute and relative enhancement of $\mathrm{CV}$ risk related to the inclusion of diabetics in a trial. Conversely, these algorithms can be used to estimate the proportion of diabetics to be included when designing a prospective study, in order to achieve a given number of CV events.

Major guidelines recognize a higher risk of CHD in DM patients, even in situations of primary prevention, as compared to non-diabetic subjects. The events rates in the comparator arms of randomized controlled trials and the meta-analyses of key statin trials show that $\mathrm{CHD}$ risk from hypercholesterolemia in non-diabetic 


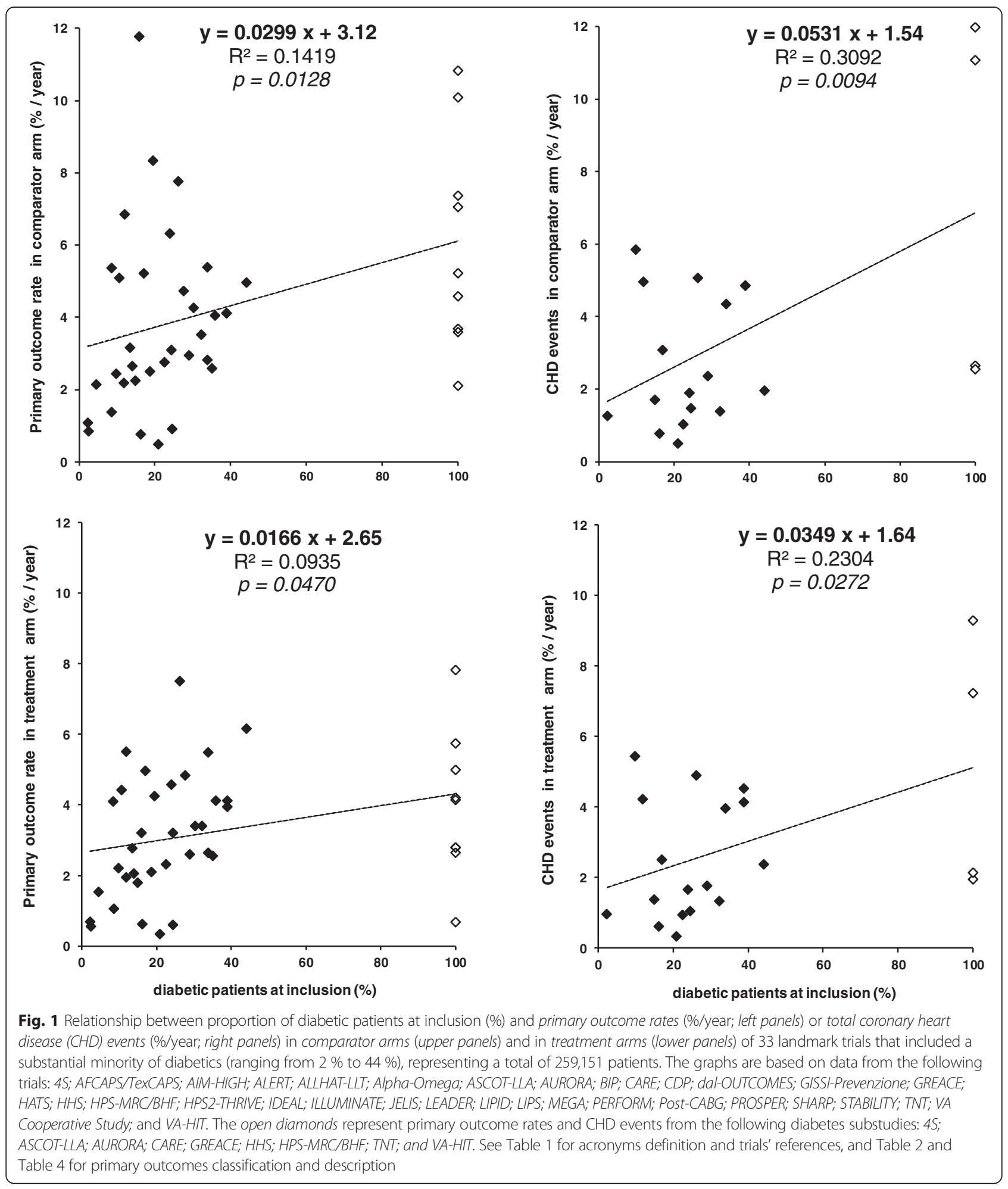

patients is proportional to baseline LDL-C level. This is also the case for type 2 DM patients, with the additional aggravating fact that this linear relationship was shifted upward compared to non-diabetics. This underlies current recommendations for effective lowering of
LDL-C as the major modifiable lipid risk factor for CHD in diabetic patients.

It should be noted that mean $\mathrm{PO}$ rate in studies focusing on diabetes was considerably lower (-46\%) than the risk that would be determined for diabetics if included, 
as a subgroup, in a clinical trial not focusing on diabetes. This follows from the fact that studies focusing on diabetes had a lower CV risk at inclusion, as well as lesser $\mathrm{PO}$ or CHD events during the study. As a result, the impact of DM on CV events must be qualified according to whether it is evaluated from diabetic subgroups of cohorts followed in cardiology (mostly in a macrovascular setting), or whether it is obtained in patients from clinical trials focusing on nutrition or diabetes (usually dealing with glycemic control or microvascular risk reduction). In addition, variation in residual risk related to T2DM in key trials may result from inhomogeneity in inclusion criteria; varying baseline $\mathrm{CV}$ risk; individual differences in diabetes duration or severity; and heterogeneous RFs exposure among diabetics.

As opposed to what occurs in microvessels, and unlike a widely held view about it, residual risk targeting large vessels is related to a limited extent only by hyperglycaemia in (pre)diabetes states. Rather, the accrued macrovascular risk is associated with the common form of T2DM (that is to say the one that expresses a MetS phenotype, including insulin resistance and hyperinsulinemia). The common pathogenic factors underlying the observed association between hyperglycemia and $\mathrm{CHD}$ are involved either $(i)$ at the onset of diabetes (promoting B-cell decompensation or altering one or two variable(s) of the hyperbolic product between insulin secretion and insulin sensitivity), and/or (ii) because they embody cardiometabolic comorbidities that increase the macrovascular risk regardless of glucose levels.

It should be noted that the slopes of the relationships between $\mathrm{CV}$ events and percentage of included diabetics were less marked when it came to comparing PO vs. CHD events rates, both in comparator and treatment arms, on one hand, or when it came to comparing PO or CHD events rates in treated arms vs. comparator arms, on the other hand. These observations suggest $(i)$ that the presence of diabetes at baseline has less adverse effect on the occurrence of certain constituents of the PO, such as allcause deaths or coronary revascularization; and (ii) that diabetic patients derive more benefits from the different treatment approaches studied than non-diabetic patients as regards the occurrence of macrovascular events [91]. In this meta-analysis, we have not distinguished between studies on the basis of pharmacological or nutritional interventions, since we based our findings on patients from comparator arms, usually receiving a placebo or standard care. When comparing less recent (published $<2005$ ) and more contemporary studies (published $\geq 2005$ ), a decrease in absolute and relative events rates was observed ( $-28 \%$ and $-1 \%$ respectively), suggestive of a reduction in exposure to CV RFs over time and/or of improved overall $\mathrm{CV}$ management. Such changes were however not significant and further, diabetic patients benefited less from this trend, reducing the absolute and relative rates by only $-14 \%$ and $-0.7 \%$. It seemed therefore appropriate to include all studies in this analysis regardless of publication year.

It is noteworthy that the increased risk of $\mathrm{CV}$ events due to the presence of a subgroup of diabetics had a pretty similar slope, whatever the $\mathrm{CV}$ risk category at baseline. It follows that the excess $\mathrm{CV}$ risk associated with the inclusion of people with diabetes in a lipidmodifying trial is relatively independent of study design, expanding the applicability of equations derived from this meta-analysis. There exists a positive relationship between biomarkers and occurrence of $\mathrm{CV}$ events [92]; our meta-analysis suggests that documenting the frequency of enlisted T2DM patients can also be used as surrogate biomarker predicting a non-modifiable component of residual CV risk. Considering that our analysis focused on populations enrolled in the comparator arms of mostly LMT studies, it would be interesting to determine the impact on residual risk arising from enlistment of diabetics in clinical trials testing several interventions in primary care [93].

This study has several limitations. Firstly, the risk estimates attributed to DM were not adjusted for age or other CV RFs comorbid to T2DM and, as in all systematic collection of published data, there is always a potential bias related to publications [94]. Secondly, the adequacy of these equations to predict $\mathrm{CV}$ outcomes has not been independently validated in a prospective context. Thirdly, for reasons related to the design and reporting of individual studies, it was not feasible to derive specific equations applicable to T1DM vs. T2DM subgroups, or to newlydiagnosed vs. long-standing T2DM patients [95]. We were not able to analyze the potential influence of glycaemic control in diabetic subgroups at baseline, due to the low reporting rate of $\mathrm{HbA}_{1 \mathrm{c}}$ values [96]. Finally, we did not examine, for reasons of brevity, the relationship between diabetes prevalence and non-CHD outcomes, such as HF, which will require dedicated meta-analyses [97].

\section{Conclusion}

This study attempted to quantify the impact of diabetes on the occurrence of $\mathrm{CV}$ events during a lipid-modifying trial, based on the proportion of known diabetics included. The component of absolute and relative residual $\mathrm{CV}$ risk associated with diabetes can be measured from linear equations relating diabetes prevalence to primary outcomes or CHD rates. Such calculations may help clinical study designers when selecting inclusion criteria; cohort size; and planned diabetics' enrollment, so as to achieve sufficient $\mathrm{CV}$ events over time.

\section{Abbreviations}

apoB: apolipoprotein $B_{100}$; BSR: Between-study range; CETP: Cholesteryl ester transfer protein; CHD: Coronary heart disease; CV: Cardiovascular;

DM: Diabetes mellitus; DSS: Diabetes substudy; $\mathrm{HbA}_{1}$ : glycated hemoglobin; 
HDL: High-density lipoprotein; HDL-C: High-density lipoprotein cholesterol; LDL: Low-density lipoproteins; LDL-C: Low-density lipoprotein cholesterol; Lp-PLA2: Lipoprotein-associated phospholipase A2; non-HDL-C: non-highdensity lipoprotein cholesterol; NS: Non-significant; PO: Primary outcome; PP: Primary prevention; PPAR: Peroxisome proliferator-activated receptor; RF: Risk factor; SD: Standard deviation; SP: Secondary prevention; T1DM: Type 1 diabetes mellitus; T2DM: Type 2 diabetes mellitus; TC: Total cholesterol; TG: Triglycerides (triacylglycerols)

\section{Competing interests}

The authors declare that they have no competing interests.

\section{Authors' contributions}

All authors contributed equally to the manuscript. M.P.H., S.A.A. and M.F.R. designed the study, set up and manage the database, and performed the statistical analyses. E.B and K.D.A. participated in study design development and helped to draft the manuscript. All authors read and approved the final manuscript.

\section{Acknowledgements}

This study received no financial support.

\section{Author details}

'Division of Endocrinology \& Nutrition, Cliniques universitaires St-Luc and Institut de Recherche Expérimentale et Clinique (IREC), Université catholique de Louvain, Brussels, Belgium. ${ }^{2}$ Service de Maladies Métaboliques et Endocriniennes, Centre Hospitalier et Universitaire de Brazzaville, Brazzaville, Congo. ${ }^{3}$ Service d'Endocrinologie et Métabolisme, CNHU HKM Cotonou, Université d'Abomey-Calavi, Abomey-Calavi, Bénin. ${ }^{4}$ Division of Cardiology, Cliniques universitaires St-Luc and Pôle de Recherche Cardiovasculaire, Institut de Recherche Expérimentale et Clinique (IREC), Université catholique de Louvain, Brussels, Belgium.

\section{Received: 2 March 2015 Accepted: 6 May 2015}

Published online: 21 May 2015

\section{References}

1. Hermans MP, Ahn SA, Rousseau MF. Effect of lipid management on coronary heart disease risk in patients with diabetes. In: McGuire DK, Nikolaus M, editors. Diabetes in Cardiovascular Disease. A Companion to Braunwald's Heart Disease. Philadelphia: Elsevier Saunders; 2015. p. 181-202.

2. Haffner SM, Lehto S, Rönnemaa T, Pyörälä K, Laakso M. Mortality from coronary heart disease in subjects with type 2 diabetes and in non-diabetic subjects with and without prior myocardial infarction. N Engl J Med. 1998;339:229-34

3. Juutilainen A, Lehto S, Rönnemaa T, Pyörälä K, Laakso M. Type 2 diabetes as a 'coronary heart disease equivalent'. An 18-year prospective populationbased study in Finnish subjects. Diabetes Care. 2005;28:2901-7.

4. Buyken AE, von Eckardstein A, Schulte H, Cullen P, Assmann G. Type 2 diabetes mellitus and risk of coronary heart disease: results of the 10-year follow-up of the PROCAM study. Eur J Cardiovasc Prev Rehabil. 2007;14:230-6.

5. CTT-Cholesterol treatment trialists' (CTT) Collaborators. Efficacy of cholesterollowering therapy in 18686 people with diabetes in 14 randomised trials of statins: a meta-analysis. Lancet. 2008;371:117-25.

6. Mazzone T, Chait A, Plutzky J. Cardiovascular disease risk in type 2 diabetes mellitus: insights from mechanistic studies. Lancet. 2008;371:1800-9.

7. Schramm TK, Gislason GH, Køber L, Rasmussen S, Rasmussen JN, Abildstrøm SZ, et al. Diabetes patients requiring glucose-lowering therapy and nondiabetics with a prior myocardial infarction carry the same cardiovascular risk: a population study of 3.3 million people. Circulation. 2008;117:1945-54.

8. Fruchart JC, Sacks FM, Hermans MP, Assmann G, Brown WV, Ceska R, et al. Residual Risk Reduction Initiative (R3I). The Residual Risk Reduction Initiative: a call to action to reduce residual vascular risk in dyslipidaemic patient. Diab Vasc Dis Res. 2008;5:319-35.

9. Hermans MP, Ahn SA, Rousseau MF. Residual vascular risk in T2DM: the next frontier. In: Mark B, editor. Recent Advances in the Pathogenesis, Prevention and Management of Type 2 Diabetes and its Complications. Croatia: Zimering, Intech, Rijeka; 2011. p. 45-66.

10. Fruchart JC, Davignon J, Hermans MP, Al-Rubeaan K, Amarenco P, Assmann $\mathrm{G}$, et al. Residual Risk Reduction Initiative (R3i). Residual macrovascular risk in 2013: what have we learned? Cardiovasc Diabetol. 2014;13:26
11. Wanner C, Krane V, März W, Olschewski M, Mann JF, Ruf G, et al. Atorvastatin in patients with type 2 diabetes mellitus undergoing hemodialysis. N Engl J Med. 2005;353:238-48.

12. Scandinavian Simvastatin Survival Study Group. Randomised trial of cholesterol lowering in 4444 patients with coronary heart disease: the Scandinavian Simvastatin Survival Study (4S). Lancet. 1994;344:1383-9.

13. Kjekshus J, Pedersen TR, For the Scandinavian Simvastatin Survival Study Group. Reducing the risk of coronary events: Evidence from the Scandinavian Simvastatin Survival Study (4S). Am J Cardiol. 1995;76:64C-8.

14. Pyorala K, Pedersen TR, Kjekshus J, Faergeman O, Olsson AG, Thorgeirsson G. the 4S Study Group: Cholesterol lowering with simvastatin improves prognosis of diabetic patients with coronary heart disease. A subgroup analysis of the Scandinavian Simvastatin Survival Study (4S). Diabetes Care. 1997;20:614-20.

15. The ACCORD Study Group. Action to control cardiovascular risk in diabetes (ACCORD) trial: Design and Methods. Am J Cardiol. 2007;99:21j-33.

16. The ACCORD Study Group. Effects of combination lipid therapy in type 2 diabetes mellitus. N Engl J Med. 2010;362:1563-74.

17. Griffin SJ, Borch-Johnsen K, Davies MJ, Khunti K, Rutten GE, Sandbæk A, et al. Effect of early intensive multifactorial therapy on 5-year cardiovascular outcomes in individuals with type 2 diabetes detected by screening (ADDITION-Europe): a cluster-randomised trial. Lancet. 2011;378:156-67.

18. Van Den Donk Van Den Donk M, Griffin SJ, Stellato RK, Simmons RK, Sandbæk A, Lauritzen T, et al. Effect of early intensive multifactorial therapy compared with routine care on self-reported health status, general well-being, diabetesspecific quality of life and treatment satisfaction in screen-detected type 2 diabetes mellitus patients (ADDITION-Europe): a cluster-randomised trial. Diabetologia. 2013;56:2367-77.

19. Downs JR, Beere PA, Whitney E, Clearfield M, Weis S, Rochen J, et al. Design $\&$ rationale of the Air Force/Texas coronary atherosclerosis prevention study (AFCAPS/TexCAPS). Am J Cardiol. 1997;80:287-93.

20. Downs JR, Clearfield M, Weis S, Whitney E, Shapiro DR, Beere PA, et al. Primary prevention of acute coronary events with lovastatin in men and women with average cholesterol levels. Results from AFCAPS/TexCAPS. JAMA. 1998;279:1615-22.

21. The AIM-HIGH Investigators. The role of niacin in raising high-density lipoprotein cholesterol to reduce cardiovascular events in patients with atherosclerotic cardiovascular disease and optimally treated low-density lipoprotein cholesterol: Baseline characteristics of study participants. The atherothrombosis intervention in metabolic syndrome with low HDL/high triglycerides: Impact on global health outcomes (AIM-HIGH) trial. Am Heart J. 2011;161:538-43.

22. AIM-HIGH Investigators, Boden WE, Probstfield JL, Anderson T, Chaitman BR, Desvignes-Nickens $P$, et al. Niacin in patients with low HDL cholesterol levels receiving intensive statin therapy. N Engl J Med. 2011;365:2255-67.

23. Lincoff AM, Tardif JC, Neal B, Nicholls SJ, Rydén L, Schwartz GG, et al. Evaluation of the dual peroxisome proliferator-activated receptor $a / \gamma$ agonist aleglitazar to reduce cardiovascular events in patients with acute coronary syndrome and type 2 diabetes mellitus: rationale and design of the AleCardio trial. Am Heart J. 2013;166:429-34.

24. Lincoff AM, Tardif JC, Schwartz GG, Nicholls SJ, Rydén L, Neal B, et al. Effect of aleglitazar on cardiovascular outcomes after acute coronary syndrome in patients with type 2 diabetes mellitus: the AleCardio randomized clinical trial. JAMA. 2014:311:1515-25.

25. Holdaas H, Fellström B, Jardine AG, Holme I, Nyberg G, Fauchald P, et al. Effect of fluvastatin on cardiac outcomes in renal transplant recipients: a multicentre, randomised, placebo-controlled trial. Lancet. 2003;361:2024-31.

26. The ALLHAT Officers and Coordinators for the ALLHAT Collaborative Research Group. Major outcomes in moderately hypercholesterolemic, hypertensive patients randomized to pravastatin vs usual care: The antihypertensive and lipid-lowering treatment to prevent heart attack trial (ALLHAT-LLT). JAMA. 2002;288:2998-3007.

27. Kromhout D, Giltay EJ, Geleijnse JM. Alpha Omega Trial Group. n-3 fatty acids and cardiovascular events after myocardial infarction. N Engl J Med. 2010;363:2015-26

28. Sever PS, Dahlöf B, Poulter NR, Wedel H, Beevers G, Caulfield M, et al. Prevention of coronary and stroke events with atorvastatin in hypertensive patients who have average or lower-than-average cholesterol concentrations, in the Anglo-Scandinavian Cardiac Outcomes Trial-Lipid Lowering Arm (ASCOTT-LLA): a multicentre randomised controlled trial. Lancet. 2003:361:1149-58. 
29. Sever PS, Poulter NR, Dahlöf B, Wedel H, Collins R, Beevers G, et al. Reduction in cardiovascular events with atorvastatin in 2532 patients with type 2 diabetes. Anglo-Scandinavian Cardiac Outcomes Trial-Lipid Lowering Arm (ASCOTT-LLA). Diabetes Care. 2005:28:1151-7.

30. Knopp RH, D'Emden M, Smilde JG, Pocock SJ, The ASPEN Study Group. Efficacy and safety of atorvastatin in the prevention of cardiovascular end points in subjects with type 2 diabetes. The atorvastatin study for prevention of coronary heart disease endpoints in non-insulin-dependent diabetes mellitus (ASPEN). Diabetes Care. 2006;29:1478-85.

31. Felström BC, Jardine AG, Schmeider RE, Holdaas H, Bannister K, Beutler J, et al. Rosuvastatin and cardiovascular events in patients undergoing hemodialysis. N Engl J Med. 2009;360:1395-407.

32. Holdaas H, Holme I, Schmeider RE, Jardine AG, Zannad F, Norby GE, et al. Rosuvastatin in diabetic hemodialysis patients. J Am Soc Nephrol. 2011;22:1335-41.

33. The BIP, Group S. Secondary prevention by raising HDL cholesterol and reducing triglycerides in patients with coronary artery disease. The Bezafibrate Infarction Prevention (BIP) Study. Circulation. 2000;102:21-7

34. Goldenberg I, Boyko V, Tennenbaum A, Tanne D, Behar S, Guetta V. Long-term benefit of high-density lipoprotein cholesterol-raising therapy with bezafibrate. 16-year mortality follow-up of the Bezafibrate Infarction Prevention Trial. Arch Intern Med. 2009;169:508-14.

35. Colhoun HM, Betteridge DJ, Durrington PN, Hitman GA, Neil HA Livingstone SJ, et al. Primary prevention of cardiovascular disease with atorvastatin in type 2 diabetes in the Collaborative Atorvastatin Diabetes Study (CARDS): multicentre randomised placebo-controlled trial. Lancet. 2004:364:685-96.

36. Sacks FM, Pfeffer MA, Moye LA, Rouleau JL, Rutherford JD, Cole TG, et al. The effect of pravastatin on coronary events after myocardial infarction in patients with average cholesterol levels. N Engl J Med. 1996;335:1001-9.

37. Lewis SJ, Sacks FM, Mitchell JS, East C, Glasser S, Kell S, et al. Effect of pravastatin on cardiovascular events in women after myocardial infarction: The Cholesterol and Recurrent Events (CARE) Trial. J Am Coll Cardiol. 1998;32:140-6

38. Goldberg RB, Mellies MJ, Sacks FM, Moyé LA, Howard BV, Howard WJ, et al Cardiovascular events and their reduction with pravastatin in diabetic and glucose-intolerant myocardial infarction survivors with average cholesterol levels. Subgroup analyses in the Cholesterol And Recurrent Events (CARE) Trial. Circulation. 1998:98:2513-9.

39. The Coronary Drug Project Research Group. Clofibrate and niacin in coronary heart disease. JAMA. 1975;231:360-81.

40. Canner PL, Berge KG, Wenger NK, Stamler J, Friedman L, Prineas RJ, et al. Fifteen year mortality in Coronary Drug Project patients: long-term benefit with niacin. J Am Coll Cardiol. 1986;8:1245-55.

41. Schwartz GG, Olsson AG, Ballantyne CM, Barter PJ, Holme IM, Kallend D, et al. dal-OUTCOMES Committees and Investigators. Rationale and design of the dal-OUTCOMES trial: efficacy and safety of dalcetrapib in patients with recent acute coronary syndrome. Am Heart J. 2009:158:896-901.

42. Schwartz GG, Olsson AG, Abt M, Ballantyne CM, Barter PJ, Brumm J, et al dal-OUTCOMES Investigators: Effects of dalcetrapib in patients with a recent acute coronary syndrome. New Engl J Med. 2012;367:2089-99.

43. Hanefeld M, Fischer S, Schmechel H, Rothe G, Schulze J, Dude H, et al. Diabetes Intervention Study. Multi-Intervention Trial in newly diagnosed NIDDM. Diabetes Care. 1991;14:308-17.

44. The FIELD Study Investigators. Fenofibrate intervention and event lowering in diabetes (FIELD) study: baseline characteristics and short-term effects of fenofibrate [ISRCTN64783481]. Cardiovasc Diabetol. 2005:4:13.

45. The FIELD Study Investigators. Effects of long-term fenofibrate therapy on cardiovascular events in 9795 people with type 2 diabetes mellitus (the FIELD study): randomised controlled trial. Lancet. 2005;366:1849-61.

46. Scott R, O'Brien R, Fulcher G, Pardy C, D'Emden M, Tse D, et al. Effects of fenofibrate treatment on cardiovascular disease risk in 9795 individuals with type 2 diabetes and various components of the metabolic syndrome. The Fenofibrate Intervention and Event Lowering in Diabetes (FIELD) study. Diabetes Care. 2009:32:493-8.

47. GISSI. Prevenzione Investigators (Gruppo Italiano per lo Studio della Soprovvivenza nell'Infarto Miocardico): Results of the low-dose (20 mg) pravastatin GISSI Prevenzione trial in 4271 patients with recent myocardial infarction: do stopped trials contribute to overall knowledge? Ital Heart J. 2000;1:810-20
48. Athyros VG, Papageorgiou AA, Mercouris BR, Athyrou W, Symeonidis AN, Basayannis EO, et al. Treatment with atorvastatin to the National Cholesterol Educational Program goal versus "usual" care in secondary coronary heart disease prevention. The GREek Atorvastatin and Coronary-heart-disease Evaluation (GREACE) study. Curr Med Res Opin. 2002;18:220-8.

49. Athyros VG, Papageorgiou AA, Symeonidis AN, Didangelos TP, Pehlivanidis AN, Bouloukos VI, et al. Early benefit from structured care with atorvastatin in patients with coronary heart disease and diabetes mellitus. A subgroup analysis of the GREACE Study. Angiology. 2003;54:679-90.

50. Brown BG, Zhao XQ, Chait A, Fisher LD, Cheung MC, Morse JS, et al. Simvastatin and niacin, antioxidant vitamins, or the combination for the prevention of coronary disease. N Engl J Med. 2001;345:1583-92.

51. Frick MH, Elo O, Haapa K, Heinonen OP, Heinsalmi P, Helo P, et al. Primaryprevention trial with gemfibrozil in middle-aged men with dyslipidemia: Safety of treatment, changes in risk factors, and incidence of coronary heart disease. N Engl J Med. 1987;317:1237-45.

52. Koskinen $\mathrm{P}$, Mänttäri $\mathrm{M}$, Manninen $\mathrm{V}$, Huttunen JK, Heinonen $\mathrm{OP}$, Frick $\mathrm{MH}$. Coronary heart disease incidence in NIDDM patients in the Helsinki Heart Study. Diabetes Care. 1992:15:820-5.

53. Heart Protection Study Collaborative Group. MRC/BHF Heart Protection Study of cholesterol lowering with simvastatin in 20536 high-risk individuals: a randomised placebo-controlled trial. Lancet. 2002;360:7-22.

54. Heart Protection Study Collaborative Group. MRC/BHF Heart Protection Study of cholesterol lowering with simvastatin in 5963 people with diabetes: a randomised placebo-controlled trial. Lancet. 2003;361:2005-16.

55. HPS2-THRIVE Collaborative Group. HPS2-THRIVE randomized placebocontrolled trial in 25673 high-risk patients of ER niacin/laropiprant: trial design, pre-specified muscle and liver outcomes, and reasons for stopping study treatment. Eur Heart J. 2013;34:1279-91.

56. Pedersen TR, Faergeman $\mathrm{O}$, Kastelein JJP, Olsson AG, Tikkanen MJ, Holme I, et al. Design and baseline characteristics of the incremental decrease in end points through aggressive lipid lowering study. Am J Cardiol. 2004;94:720-4

57. Pedersen TR, Faergeman O, Kastelein JJP, Olsson AG, Tikkanen MJ, Holme I, et al. High-dose atonastatin vs usual-dose simvastatin for secondary prevention after myocardial infarction. The IDEAL Study: a randomised controlled trial. JAMA. 2005;294:2437-45.

58. Barter PJ, Caulfield M, Eriksson M, Grundy SM, Kastelein JJ, Komajda M, et al. ILLUMINATE Investigators: Effects of torcetrapib in patients at high risk for coronary events. N Engl J Med. 2007;357:2109-22.

59. Yokoyama M, Origasa H, Matsuzaki M, Matsuzawa Y, Saito Y, Ishikawa Y, et al. Japan EPA lipid intervention study (JELIS) Investigators: Effects of eicosapentaenoic acid on major coronary events in hypercholesterolaemic patients (JELIS): a randomized open-label, blinded endpoint analysis. Lancet. 2007:369:1090-8.

60. Meade TW. The MRC General Practice Research Framework and Participating Vascular Clinics: Design and intermediate results of the Lower Extremity Arterial Disease Event Reduction (LEADER) trial of bezafibrate in men with lower extremity arterial disease. Curr Control Trials Cardiovasc Med. 2001;2:195-204.

61. Meade T, Zuhrie R, Cook C. Cooper J on behalf of MRC General Practice Research Framework: Bezafibrate in men with lower extremity arterial disease: randomised controlled trial. BMJ. 2002;325:1139.

62. The Lipid Study Group. Design features and baseline characteristics of the LIPID (Long-Term Intervention With Pravastatin in Ischemic Disease) Study: a randomized trial in patients with previous acute myocardial infarction and/ or unstable angina pectoris. Am J Cardiol. 1995;76:474-9.

63. The Lipid Study Group. Prevention of cardiovascular events and death with pravastatin in patients with coronary heart disease and a broad range of initial cholesterol levels. N Engl J Med. 1998;339:1349-57.

64. The Lipid Study Group. Long-term effectiveness and safety of pravastatin in 9014 patients with coronary heart disease and average cholesterol concentrations: the LIPID trial follow-up. Lancet. 2002;359:1379-87.

65. Serruys PW, de Feyter P, Macaya C, Kokott N, Puel J, Vrolix M, et al. Fluvastatin for prevention of cardiac events following successful first percutaneous coronary intervention. A randomized controlled trial. JAMA. 2002;287:3215-22

66. Nakamura H, Arakawa K, Itakura H, Kitabatake A, Goto Y, Toyota T, et al. Primary prevention of cardiovascular disease with pravastatin in Japan (MEGA Study): a prospective randomised controlled trial. Lancet. 2006:368:1155-63. 
67. Bosch J, Gerstein HC, Dagenais GR, Díaz R, Dyal L, Jung $H$, et al. For the ORIGIN Trial Investigators. n-3 fatty acids and cardiovascular outcomes in patients with dysglycemia. N Engl J Med. 2012;367:309-18.

68. Bousser MG, Amarenco P, Chamorro A, Fisher M, Ford I, Fox KM, et al. PERFORM Study Investigators. Terutroban versus aspirin in patients with cerebral ischaemic events (PERFORM): a randomised, double-blind, parallelgroup trial. Lancet. 2011;377:2013-22.

69. The Post Coronary Artery Bypass Graft Trial Investigators. The effect of aggressive lowering of low-density lipoprotein cholesterol levels and low-dose anticoagulation on obstructive changes in saphenous-vein coronary-artery bypass grafts. N Engl J Med. 1997;336:153-62.

70. Knatterud GL, Rosenberg Y, Campeau L, Geller NL, Hunninghake DB, Forman SA, et al. Long-term effects on clinical outcomes of aggressive lowering of lowdensity lipoprotein cholesterol levels and low-dose anticoagulation in the Post Coronary Artery Bypass Graft Trial. Circulation. 2000;102:157-65.

71. Estruch R, Ros E, Salas-Salvadó J, Covas MI, Corella D, Arós F, et al. PREDIMED Study Investigators. Primary prevention of cardiovascular disease with a Mediterranean diet. N Engl J Med. 2013;368:1279-90.

72. Charbonnel B, Dormandy J, Erdmann E, Massi-Benedetti M, Skene A, The PROActive Study Group. The Prospective Pioglitazone Clinical Trial in Macrovascular Events (PROactive). Diabetes Care. 2004:27:1647-53.

73. Dormandy JA, Charbonnel B, Eckland DJA, Erdmann E, Massi-Benedetti M, Moules IK, et al. Secondary prevention of macrovascular events in patients with type 2 diabetes in the PROactice Study (PROspective pioglitAzone Clinical Trial In macro Vascular Events): a randomised controlled trial. Lancet. 2005;366:1279-89.

74. Yoshii H, Onuma T, Yamazaki T, Watada H, Matsuhisa M, Matsumoto M, et al. Effects of pioglitazone on macrovascular events in patients with type 2 diabetes mellitus at high risk of stroke: the PROFIT-J study. J Atheroscler Thromb. 2014;21:563-73.

75. Shepherd J, Blauw GJ, Murphy MB, Bollen EL, Buckley BM, Cobbe SM, et al. Pravastatin in elderly individuals at risk of vascular disease (PROSPER): a randomised controlled trial. Lancet. 2002;360:1623-30.

76. Rischio and Prevenzione Investigators. Efficacy of n-3 polyunsaturated fatty acids and feasibility of optimizing preventive strategies in patients at high cardiovascular risk: rationale, design and baseline characteristics of the Rischio and Prevenzione study, a large randomised trial in general practice. Trials. 2010;11:68.

77. Risk and Prevention Study Collaborative Group, Roncaglioni MC, Tombesi M, Avanzini F, Barlera S, Caimi V, et al. n-3 fatty acids in patients with multiple cardiovascular risk factors. N Engl J Med. 2013;368:1800-8.

78. Baigent C, Landray MJ, Reith C, Emberson J, Wheeler DC, Tomson C, et al. The effects of lowering LDL cholesterol with simvastatin plus ezetimibe in patients with chronic kidney disease (Study of Heart and Renal Protection): a randomised placebo-controlled trial. Lancet. 2011;377:2181-92.

79. White H, Held C, Stewart R, Watson D, Harrington R, Budaj A, et al. Study design and rationale for the clinical outcomes of the STABILITY Trial (STabilization of Atherosclerotic plaque By Initiation of darapLadlb TherapY) comparing darapladib versus placebo in patients with coronary heart disease. Am Heart J. 2010;160:655-61.

80. STABILITY Investigators, White HD, Held C, Stewart R, Tarka E, Brown R, et al. Darapladib for preventing ischemic events in stable coronary heart disease. N Engl J Med. 2014;370:1702-11.

81. Gaede $\mathrm{P}$, Lund-Andersen $\mathrm{H}$, Parving $\mathrm{HH}$, Pedersen O. Effects of a multifactorial intervention on mortality in type 2 diabetes. N Engl J Med. 2008;358:580-91.

82. Waters DD, Guyton JR, Herrington DM, McGowan MP, Wenger NK, Shear C, et al. Treating to new targets (TNT) study: Does lowering low-density lipoprotein cholesterol levels below currently recommended guidelines yield incremental clinical benefit? Am J Cardiol. 2004;93:145-8.

83. LaRosa JC, Grundy SM, Waters DD, Shear C, Barter P, Fruchart JC, et al. Intensive lipid lowering with atorvastatin in patients with stable coronary disease. N Engl J Med. 2005;352:1425-35.

84. Shepherd J, Kastelein JJP, Bittner V, Deedwania P, Breazna A, Dobson S, et al. Effect on intensive lipid lowering with atorvastatin on renal function in patients with coronary heart disease: The Treating to New Targets (TNT) Study. Clin J Am Soc Nephrol. 2007;2:1131-9.

85. Barter P, Gotto AM, LaRosa JC, Maroni J, Szarek M, Grundy SM, et al. HDL cholesterol, very low levels of LDL cholesterol, and cardiovascular events. N Engl J Med. 2007;357:1301-10.

86. Shepherd J, Barter P, Carmena R, Deedwania P, Fruchart JC, Haffner S, et al. Effect of lowering LDL cholesterol substantially below currently recommended levels in patients with coronary heart disease and diabetes. The Treating to New Targets (TNT) Study. Diabetes Care. 2006;29:1220-6.

87. The Veterans Administration Cooperative Study Group. The treatment of cerebrovascular disease with clofibrate. Final report of the Veterans Administration Cooperative Study of Atherosclerosis, Neurology Section. Stroke. 1973;4:684-93.

88. Rubins HB, Robins SJ, Collins D, Fye CL, Anderson JW, Elam MB, et al. Gemfibrozil for the secondary prevention of coronary heart disease in men with low levels of high-density lipotrotein cholesterol. N Engl J Med. 1999:341:410-8.

89. Robins SJ, Collins D, Wittes JT, Papademetriou V, Deedwania PC, Schaefer EJ, et al. Relation of gemfibrozil treatment and lipid levels with major coronary events. VA-HIT: a randomized controlled trial. JAMA. 2001;285:1585-91.

90. Rubins HB, Robins SJ, Collins D, Nelson DB, Elam MB, Schaefer EJ, et al. Diabetes, plasma insulin, and cardiovascular disease. Subgroup analysis from the Department of Veterans Affairs High-Density Lipoprotein Intervention Trial (VA-HIT). Arch Intern Med. 2002;162:2597-604.

91. Costa J, Borges M, David C, Vaz Carneiro A. Efficacy of lipid lowering drug treatment for diabetic and non-diabetic patients: meta-analysis of randomised controlled trials. BMJ. 2006;332:1115-8.

92. Ofstad AP, Gullestad L, Orvik E, Aakhus S, Endresen K, Ueland T, et al. Interleukin-6 and activin A are independently associated with cardiovascular events and mortality in type 2 diabetes: the prospective Asker and Bærum Cardiovascular Diabetes (ABCD) cohort study. Cardiovasc Diabetol. 2013;12:126.

93. Jiao FF, Fung CS, Wong CK, Wan YF, Dai D, Kwok R, et al. Effects of the Multidisciplinary Risk Assessment and Management Program for Patients with Diabetes Mellitus (RAMP-DM) on biomedical outcomes, observed cardiovascular events and cardiovascular risks in primary care: a longitudinal comparative study. Cardiovasc Diabetol. 2014;13:127.

94. Wang Y, Lammi-Keefe CJ, Hou L, Hu G. Impact of low-density lipoprotein cholesterol on cardiovascular outcomes in people with type 2 diabetes: a meta-analysis of prospective cohort studies. Diabetes Res Clin Pract. 2013;102:65-75.

95. Rousan TA, Pappy RM, Chen AY, Roe MT, Saucedo JF. Impact of diabetes mellitus on clinical characteristics, management, and in-hospital outcomes in patients with acute myocardial infarction (from the NCDR). Am J Cardiol. 2014;114:1136-44.

96. Fatemi O, Yuriditsky E, Tsioufis C, Tsachris D, Morgan T, Basile J, et al. Impact of intensive glycemic control on the incidence of atrial fibrillation and associated cardiovascular outcomes in patients with type 2 diabetes mellitus (from the Action to Control Cardiovascular Risk in Diabetes Study). Am J Cardiol. 2014;114:1217-22.

97. Sarma S, Mentz RJ, Kwasny MJ, Fought AJ, Huffman M, Subacius H, et al. EVEREST investigators. Association between diabetes mellitus and postdischarge outcomes in patients hospitalized with heart failure: findings from the EVEREST trial. Eur J Heart Fail. 2013;15:194-202.

\section{Submit your next manuscript to BioMed Central and take full advantage of:}

- Convenient online submission

- Thorough peer review

- No space constraints or color figure charges

- Immediate publication on acceptance

- Inclusion in PubMed, CAS, Scopus and Google Scholar

- Research which is freely available for redistribution 